\title{
CARACTERÍSTICAS SENSORIAIS E INSTRUMENTAIS DO LEITE PASTEURIZADO INTEGRAL
}

\section{Sensory and instrumental characteristics of integral pasteurized milk}

\author{
Marlice Salete Bonacina ${ }^{I^{*}}$, Marina Andréa Baccin ${ }^{1}$, Leonardo Souza da Rosa
}

\begin{abstract}
RESUMO
O objetivo deste estudo foi determinar o perfil sensorial do leite pasteurizado integral, e relacionar os resultados sensoriais e instrumentais. Foram coletadas, aleatoriamente, cinco amostras de marcas diferentes de leite pasteurizado integral (marcas A, B, C, D e E), comercializadas no município de Erechim, RS, das quais duas eram homogeneizadas e três não homogeneizadas. As medidas de cor instrumental foram realizadas através da escala CIELAB ( $\left.\mathrm{L}^{*}, \mathrm{a}^{*}, \mathrm{~b}^{*}\right)$. Os atributos sensoriais foram avaliados através da análise descritiva de perfil livre e o teste de aceitação foi realizado por 44 consumidores utilizando uma escala hedônica hibrida de $9 \mathrm{~cm}$. A partir dos resultados foi possível verificar que as amostras de leite que foram submetidas a etapa de homogeneização, durante o processo de fabricação do leite pasteurizado, apresentaram uma coloração mais branca e uma aparência mais homogênea, quando comparada com as amostras de leite que não foram submetidas a homogeneização. No entanto, estes termos sensoriais não influenciaram na aceitação dos leites, uma vez que a amostra que apresentou menor valor médio $(3,8)$ para aceitação foi a marca de leite submetida a etapa de homogeneização. Através da análise da relação obtida entre os resultados das variáveis estudadas, foi possível observar uma forte correlação entre as medidas sensoriais e instrumentais realizadas nas diferentes amostras de leite, demonstrando a importância da realização da correlação entre estas medidas para a indústria de alimentos e pesquisadores, uma vez que as análises sensoriais descritivas são obtidas com maior demanda de tempo e custo.
\end{abstract}

Palavras-chave: leite homogeneizado; colorímetro; correlação.

1 Instituto Federal de Educação Ciência e Tecnologia do Rio Grande do Sul (IFRS), Rua Domingos Zanella, 104, Bairro Três Vendas, 99713-028, Erechim, RS, Brasil. E-mail: marlice.bonacina@ erechim.ifrs.edu.br

* Autor para correspondência.

Recebido / Received: $21 / 06 / 2017$

Aprovado / Approved: 08/12/2017 


\begin{abstract}
The aim of this study was to determine the sensorial profile of whole pasteurized milk, and to relate the sensorial and instrumental results. Five samples of different brands of whole pasteurized milk were randomly collected (brands A, B, C, D e E) in different supermarkets in the city of Erechim, RS, two of which were homogenized and three were not homogenized. The instrumental color measurements were performed using CIELAB $\left(\mathrm{L}^{*}, \mathrm{a}^{*}, \mathrm{~b}^{*}\right)$ scale. The sensorial attributes were evaluated through the Free Profile descriptive analysis and the acceptance test was performed by 44 consumers using a hybrid hedonic scale of $9 \mathrm{~cm}$. From the results it was possible to verify that milk samples which were submitted to homogenization during the manufacturing process of pasteurized milk showed a whiter coloration and a more homogenous appearance when compared to samples of milk that were not submitted to homogenization. However, these sensorial terms did not influence the acceptance of the milk, once the sample with the lowest mean value $(3,8)$ for acceptance was a milk brand submitted to the homogenization. By analyzing the relationship between the results of the studied variables, it was possible to observe a strong correlation between the sensorial and instrumental measurements performed in the different milk samples, demonstrating the importance of the correlation between these analytical approaches for the food industry and researchers, since the descriptive sensorial analyzes are obtained with greater demand of time and cost.
\end{abstract}

Keywords: homogenized milk, colorimeter; correlation.

\section{INTRODUÇÃO}

O Rio Grande do Sul é o segundo estado com maior produção de leite no Brasil, atrás apenas de Minas Gerais. Teve uma produção de 4,6 bilhões de litros em 2014, com aproximadamente 125 mil famílias envolvidas na atividade, das quais 85 mil são fornecedoras de leite para as indústrias. Porém, devido a competitividade do mercado, produzir não é mais suficiente. Para fazer frente à dura concorrência, onde os consumidores têm o papel principal no mercado, as indústrias devem, obrigatoriamente, investir em qualidade, iniciando pela matéria-prima (GIGANTE 2004).

Dentre os derivados lácteos podemos destacar o leite integral pasteurizado, o qual é elaborado a partir do leite cru refrigerado na propriedade rural, que apresenta as especificações de produção, de coleta e de qua- lidade dessa matéria-prima contidas em Regulamento Técnico próprio (IN 62) e que tenha sido transportado a granel até o estabelecimento processador (BRASIL, 2011).

A aplicação do tratamento térmico não garante a qualidade do leite pasteurizado, pois a existência de problemas relacionados a adulterações, condições higiênicas deficientes durante os processos de obtenção, manipulação e conservação vem sendo considerada como uma das principais razões para a perda de qualidade do produto. Por este motivo, nos últimos anos, a qualidade do leite pasteurizado vem sendo motivo de questionamentos por parte dos consumidores, órgãos fiscalizadores e regulamentadores, os quais vêm impondo modificações na legislação brasileira, visando modificar a forma de produzir leite contribuindo assim, com a melhoria da qualidade do produto (TRONCO, 2013). 
Os consumidores consideram principalmente os atributos sensoriais para definir a qualidade do leite. Porém as características sensoriais podem variar em função da origem e qualidade da matéria-prima utilizada, bem como, das diferentes tecnologias de produção aplicadas. Devido a essas diferenças, a caracterização sensorial do leite integral pasteurizado, produzido por diferentes empresas, torna-se de fundamental importância para definir o tipo de produto que o consumidor mais gosta.

Além disso, a manutenção das características sensoriais do produto contribui para a lealdade do consumidor em um mercado cada vez mais competitivo, sendo parte inerente ao plano de controle de qualidade de uma indústria (TEIXEIRA, 2009).

Entre os testes sensoriais utilizados para caracterizar sensorialmente o alimento, destaca se o perfil livre, desenvolvida por Williams et al. (1984). Essa técnica baseiase no princípio de que as pessoas percebem as mesmas características sensoriais no produto, mesmo que as expressem de forma diferenciada. É considerada uma técnica descritiva livre, ou seja, os provadores têm a liberdade de classificar a amostra de acordo com sua percepção e entendimento. Apresenta como vantagem o curto tempo para aplicação, pois emprega somente uma explanação sobre o levantamento dos atributos, o uso da escala e das fichas de avaliação (STONE; SIDEL, 2004). O número de participantes pode variar de acordo com a familiaridade com o produto, disponibilidade de tempo e experiência (KITZBERGER et al., 2010).

Dentre os atributos sensoriais, a aparência, principalmente a cor, é um parâmetro importante para a determinação da qualidade dos alimentos auxiliando no controle da matéria-prima, nas alterações causadas pelo processamento e armazenamento (MACDOUGALL, 2002). Apesar da inspeção da cor através da visualização humana ser considerada adequada, observa-se que quando ocorrem mudanças de iluminação, está análise torna-se subjetiva e variável. Desta forma segundo León et al. (2006), é recomendável que a análise também seja feita através do uso de um instrumento de medida da cor, como por exemplo, os colorímetros Minolta ou Hunter Lab.

Assim, a cor pode ser relatada por três diferentes parâmetros representados em um diagrama tridimensional a fim de definir a coloração do produto. O parâmetro $\mathrm{L}^{*}$ varia de 0 a 100, o máximo representa uma perfeita reflexão difusa, indicando a coloração branca, enquanto o valor mínimo representa coloração preta. Já os eixos $\mathrm{a}^{*}$ e b* não apresentam limites numéricos específicos. A coordenada $a^{*}$ varia do vermelho $\left(+\mathrm{a}^{*}\right)$ ao verde $\left(-a^{*}\right)$, e a coordenada $b^{*}$ do amarelo $\left(+b^{*}\right)$ ao azul $\left(-b^{*}\right)$ (HUNTERLAB, 1996; BAKKER et al., 1986).

Neste contexto, o objetivo deste estudo foi avaliar a aceitação, traçar o perfil sensorial e relacionar respostas instrumentais e sensoriais de diferentes marcas de leite integral pasteurizado.

\section{MATERIAL E MÉTODOS}

\section{Coleta das amostras}

A pesquisa apresentou uma abordagem descritiva exploratória, caracterizada como estudo de caso. Foram coletadas, aleatoriamente, cinco amostras de marcas diferentes de leite pasteurizado integral (marcas A, B, C, D e E), comercializadas no município de Erechim, RS, das quais duas eram homogeneizadas e três não homogeneizadas. As amostras de leite foram transportadas em caixas isotérmicas até o laboratório de análise sensorial do Instituto Federal de Educação, Ciência e Tecnologia do Rio Grande do Sul Campus Erechim, onde realizou-se a medida instrumental de cor e a avaliação sensorial. 


\section{Análise de cor}

As medidas de cor dos leites pasteurizados foram avaliadas através da escala
CIELAB (L*, a*, b*), utilizando um colorímetro portátil Konica Minolta, Chroma Meter CR-410, previamente calibrado e ajustado para operar com iluminante D65.

Tabela 1 - Definição dos materiais de referência para os leites pasteurizados.

\begin{tabular}{|c|c|}
\hline ATRIBUTOS & REFÊRENCIAS \\
\hline \multicolumn{2}{|l|}{ ODOR } \\
\hline Característico & Pouco: Leite diluído em 50\% de água destilada. \\
\hline Caracturistito & Muito: Leite com adição de $30 \%$ de leite em pó integral. \\
\hline Doce & Pouco: Leite diluído em 50\% de água destilada. \\
\hline Doce & Muito: Leite com adição de 2\% de açúcar. \\
\hline Azedo & Pouco: Leite diluído em 50\% de água destilada. \\
\hline & Muito: Leite com adição de $0,2 \%$ de ácido cítrico. \\
\hline Gordure & Pouco: Leite diluído em 50\% de água destilada. \\
\hline Gordura & Muito: Leite com adição de $30 \%$ de creme de leite. \\
\hline
\end{tabular}

APARENNCIA

Cor

Branco: Leite com adição de $30 \%$ de creme de leite.

Amarelado: Leite com adição de $50 \%$ de leite em pó integral.

Viscosidade

Pouco: Leite diluído em $50 \%$ de água destilada.

Muito: Leite com adição de $30 \%$ de leite em pó integral.

Pouco: Leite diluído em 50\% de água destilada.

Homogeneidade

Muito: Leite com adição de 30\% de leite em pó integral.

Opaco

Pouco: Leite com adição de $30 \%$ de leite em pó integral.

Muito: Leite com adição de $30 \%$ de creme de leite.

SABOR

Sabor característico

Pouco: Leite diluído em 50\% de água destilada.

Muito: Leite com adição de $25 \%$ de leite em pó integral.

Gosto doce

Pouco: Leite diluído em 50\% de água destilada.

Muito: Leite com adição de $1 \%$ de açúcar.

Pouco: Leite diluído em $50 \%$ de água destilada.

Sabor gordura

Muito: Leite com adição de $30 \%$ de creme de leite.

Gosto salgado

Pouco: Leite diluído em 50\% de água destilada.

Muito: Leite com adição de $0,1 \%$ de cloreto de sódio.

Pouco: Leite diluído em 50\% de água destilada.

Gosto azedo

Muito: Iogurte natural (marca Danone).

Pouco: Leite diluído em 50\% de água destilada.

Ranço

Muito: Leite com adição $50 \%$ de leite em pó no limite da validade. 
Foram obtidos os valores de luminosidade (L*), intensidade da cor vermelha (a*) e intensidade da cor amarela (b*) (FERREIRA, 2004).

\section{Avaliação sensorial}

\section{Caracterização sensorial do leite pasteurizado}

A avaliação sensorial do odor, da aparência e do sabor das diferentes amostras de leite pasteurizado foram realizadas através do método perfil livre (WILLIAMS et al., 1984).

Os julgadores que compuseram a equipe para avaliação dos leites foram selecionados a partir de 25 candidatos. O poder discriminativo dos julgadores foi avaliado através do teste triangular, utilizando-se amostras de leite pasteurizado que diferiam estatisticamente entre si ao nível de $1 \%$ de significância. Foram utilizadas três sessões e os julgadores foram selecionados com no mínimo $60 \%$ de acerto (VERRUMA-BERNARDI; DAMÁSIO, 1999).

Para o desenvolvimento da terminologia descritiva do perfil livre foi utilizado o método de rede de Kelly (KELLY, 1955). No qual os julgadores, receberam amostras de leite pasteurizado aos pares em todas as combinações, e descreviam as sensações observadas em relação a aparência, odor e sabor.

Os julgadores discutiram os termos propostos e selecionaram consensualmente os termos a serem utilizados na ficha sensorial, bem como os padrões de referência (Tabela 1).

Após, foi avaliado, em triplicata, a intensidade de cada descritor nas amostras de leite, utilizando a escala não estruturada de nove centímetros, com termos de intensidade ancorados em seus extremos, sendo o mínimo à esquerda e o máximo à direita. $\mathrm{Na}$ aplicação dos testes sensoriais, as amostras foram servidas resfriadas $\left(7^{\circ} \mathrm{C}\right)$ em copos plásticos, codificados com números de três dígitos, utilizando o delineamento em blocos completos balanceados com três repetições, usando a técnica de apresentação monádica.

\section{Aceitação do leite pasteurizado}

Foram recrutados, no Campus Erechim do Instituto Federal de Educação, Ciência e Tecnologia 44 consumidores de leite. O número de consumidores foi definido, conforme recomendação de Stone e Sidel (1993). Antes de realizar o teste sensorial, os julgadores responderam um questionário contendo questões sobre dados pessoais (idade, sexo, escolaridade) e o consumo do leite.

As amostras foram servidas a temperatura de $7{ }^{\circ} \mathrm{C}$, sendo a ordem de apresentação monódica e balanceada, em copos plásticos descartáveis e codificados, utilizando uma escala hedônica hibrida de nove (9) pontos, ancorada na região central e nos extremos com anotações verbais, sendo desgostei muitíssimo a esquerda, gostei muitíssimo a direita e indiferente ao centro (VILLANUEVA et al., 2005).

\section{Análise Estatística}

O tratamento estatístico dos dados foi efetuado utilizando o Software Estatística 6.0, no módulo de Análise de Variância Univariada (ANOVA), e o teste Tukey ao nível de $5 \%$ de probabilidade $(\mathrm{p}<0,05)$ para comparação de médias. Os demais dados estatísticos foram gerados no módulo Basic Statistics.

\section{RESULTADOS E DISCUSSÃO}

\section{Análise instrumental da cor do leite pasteurizado}

Na Tabela 2 é possível verificar as médias obtidas nas análises dos componentes de cor $L^{*}, a^{*}$ e $b^{*}$, referente aos leites das diferentes marcas utilizadas neste estudo. 
Observa-se que ocorreu diferença significativa $(\mathrm{p}<0,05)$ em relação aos três parâmetros avaliados, quando comparado os leites das diferentes marcas.

A partir dos resultados da avaliação da cor instrumental, foi possível verificar que os leites das marcas " $\mathrm{A}$ " e "B" apresentaram maiores valores de $\mathrm{L}^{*}$, indicando coloração mais branca, e menores valores de b* representando menor tonalidade amarela.

Cabe ressaltar que as amostras das marcas A e B eram leites homogeneizados, aspecto este que também pode justifica a maior intensidade da cor branca e maior homogeneidade em sua aparência, pois de acordo com Venturini et al., (2007) a homogeneização é o processo que consiste em passar o leite à pressão através de um aparelho semelhante a um coador com buracos muito pequenos, reduzindo o tamanho dos glóbulos de gordura, evitando a separação destes glóbulos.

Conforme Soeiro et al. (2011), o processo de homogeneização deve ser realizado em temperatura superior a $54{ }^{\circ} \mathrm{C}$, de maneira que toda a gordura esteja em estado líquido. Em seguida, o leite deve ser resfriado a aproximadamente $4{ }^{\circ} \mathrm{C}$ e armazenado em tanques de estocagem isotérmicos até o seu envaze.

No que se refere ao parâmetro $a^{*}$, todas as amostras apresentaram tendência para a cor verde. No entanto, as amostras que não foram submetidas a etapa de homogeneização, não apresentaram o mesmo comportamento para este parâmetro, pois a amostra E diferiu-se das amostras $\mathrm{C}$ e D. Os valores de a* obtidos para estas marcas de leite, mostram que as mesmas tendem a uma coloração mais esverdeada que as demais amostras, indicando que a etapa de homogeneização não foi o fator responsável pela diferença observada para este parâmetro quando comparada as diferentes marcas de leite.

De acordo com Jing e Kitts (2002), Acevedo et al. (2006) e Dattatreya et al. (2006) diversos fatores, como o teor de carboidratos redutores; a estrutura das partículas, contidas no alimento; a temperatura e o $\mathrm{pH}$ envolvidos no processamento de um produto lácteo, contribuem para a reação de Maillard, gerando mudança na cor. Além disso, segundo García-Pérez et al. (2005) a temperatura do tratamento térmico aplicado em produtos lácteos poderá induzir uma desestabilidade da micela de caseína, e, desta forma, ocasionar o aumento do valor de a*. SCHAFFER et al. (1992) ao estudar a cor de diferentes produtos lácteos, dentre eles o leite integral pasteurizado, obtiveram valores inferiores aos encontrados neste estudo para os parâmetros $L^{*}(86,1), a *(-2,1)$ e b* $(7,8)$.

\section{Caracterização sensorial do leite pasteurizado}

Os escores médios, da caracterização sensorial das diferentes marcas de leite pasteurizado, obtidos a partir da avaliação dos julgadores para cada atributo encontram-se na Tabela 3. Observou-se que entre as cinco marcas de leite estudadas, ocorreram diferenças estatísticas $(p<0,05)$ para a cor, homogeneidade, gosto doce e gosto azedo.

Tabela 2 - Valores médios dos componentes da cor do leite pasteurizado

\begin{tabular}{cccccc}
\hline Parâmetros & Marca A & Marca B & Marca C & Marca D & Marca E \\
\hline L $^{*}$ & $92,03^{\mathrm{a}}$ & $90,50^{\mathrm{a}}$ & $87,97^{\mathrm{b}}$ & $88,40^{\mathrm{b}}$ & $87,95^{\mathrm{b}}$ \\
$\mathrm{a}^{*}$ & $-7,52^{\mathrm{b}}$ & $-7,63^{\mathrm{b}}$ & $-8,14^{\mathrm{a}}$ & $-8,11^{\mathrm{a}}$ & $-7,38^{\mathrm{b}}$ \\
$\mathrm{b}^{*}$ & $17,20^{\mathrm{e}}$ & $18,03^{\mathrm{d}}$ & $20,97^{\mathrm{b}}$ & $20,42^{\mathrm{c}}$ & $22,31^{\mathrm{a}}$ \\
\hline
\end{tabular}

Letras iguais na mesma linha, não apresentam diferença significativa $(\mathrm{p}>0,05)$. 
No que se refere a cor do leite, verificouse que as marcas A e B apresentaram uma coloração mais branca e uma maior homogeneidade em sua aparência quando comparadas com as demais amostras $(\mathrm{p}<0,05)$. Cabe ressaltar, que na escala sensorial utilizada para a avaliação das amostras, o termo branco correspondia ao extremo esquerdo da escala e o amarelo ao extremo direito. Assim como, a homogeneidade (refere-se a presença de grumos ou partículas de gordura) o extremo esquerdo correspondia a pouco homogêneo e o direito a muito homogêneo.

Estes resultados corroboram as respostas obtidas na avaliação instrumental, para estes dois parâmetros, pois os valores do coeficiente de correlação de Pearson apresentaram uma forte relação. Obteve-se 0,96 e 0,93 , respectivamente, ao relacionar os valores de $\mathrm{L}^{*}$ com os valores de cor e homogeneidade obtidos sensorialmente. Já ao relacionar os valores de $b^{*}$ com os termos cor e homogeneidade foram obtidos 0,94 e 0,95 , respectivamente, para a correlação.
Demonstrando assim que no leite integral pasteurizado a maior intensidade da cor branca e a maior homogeneidade do produto, avaliados sensorialmente, correspondem ao maior valor de * L e ao menor valor de b*, obtidos a partir do uso do colorímetro.

Estes resultados fornecem informações de uso prático no controle, desenvolvimento e padronização dos leites comercializados, demonstrando a importância da realização de estudos que correlacionam medidas instrumentais e sensoriais, uma vez que os parâmetros instrumentais geralmente são obtidos com maior facilidade e rapidez.

Outro atributo sensorial que apresentou diferença significativa para o leite foi o gosto doce e azedo, pois os leites das marcas "A", "B" e "D" apresentaram maior intensidade para o gosto doce, enquanto a marca " $\mathrm{C}$ " apresentou maior intensidade para o gosto azedo.

A Instrução Normativa $n^{\circ} 62$, de 29 de dezembro de 2011 (BRASIL, 2011), aborda a questão sensorial descrevendo que o leite pasteurizado deve ter um aspecto líquido,

Tabela 3 - Caracterização sensorial de diferentes marcas de leite pasteurizado

\begin{tabular}{lccccc}
\hline \multicolumn{1}{c}{ Atributo } & Marca A & Marca B & Marca C & Marca D & Marca E \\
\hline Odor característico & $4,8^{\mathrm{a}}$ & $4,2^{\mathrm{a}}$ & $4,8^{\mathrm{a}}$ & $5,4^{\mathrm{a}}$ & $4,3^{\mathrm{a}}$ \\
Odor adocicado & $3,8^{\mathrm{a}}$ & $4,0^{\mathrm{a}}$ & $3,5^{\mathrm{a}}$ & $4,5^{\mathrm{a}}$ & $3,4^{\mathrm{a}}$ \\
Odor azedo & $1,9^{\mathrm{a}}$ & $1,8^{\mathrm{a}}$ & $2,8^{\mathrm{a}}$ & $1,6^{\mathrm{a}}$ & $1,8^{\mathrm{a}}$ \\
Odor gordura & $2,4^{\mathrm{a}}$ & $2,3^{\mathrm{a}}$ & $3,2^{\mathrm{a}}$ & $3,3^{\mathrm{a}}$ & $2,4^{\mathrm{a}}$ \\
Cor & $2,3^{\mathrm{b}}$ & $2,7^{\mathrm{b}}$ & $5,8^{\mathrm{a}}$ & $4,8^{\mathrm{a}}$ & $5,7^{\mathrm{a}}$ \\
Viscosidade & $4,7^{\mathrm{a}}$ & $4,3^{\mathrm{a}}$ & $4,2^{\mathrm{a}}$ & $4,1^{\mathrm{a}}$ & $4,0^{\mathrm{a}}$ \\
Homogeneidade & $6,7^{\mathrm{a}}$ & $6,9^{\mathrm{a}}$ & $3,5^{\mathrm{b}}$ & $3,6^{\mathrm{b}}$ & $3,3^{\mathrm{b}}$ \\
Opaco & $2,8^{\mathrm{a}}$ & $2,7^{\mathrm{a}}$ & $3,7^{\mathrm{a}}$ & $3,5^{\mathrm{a}}$ & $3,6^{\mathrm{a}}$ \\
Sabor característico & $5,4^{\mathrm{a}}$ & $4,9^{\mathrm{a}}$ & $5,1^{\mathrm{a}}$ & $5,2^{\mathrm{a}}$ & $4,2^{\mathrm{a}}$ \\
Gosto doce & $4,5^{\mathrm{a}}$ & $4,3^{\mathrm{a}}$ & $2,5^{\mathrm{b}}$ & $4,4^{\mathrm{a}}$ & $2,5^{\mathrm{b}}$ \\
Sabor gordura & $4,3^{\mathrm{a}}$ & $3,8^{\mathrm{a}}$ & $4,5^{\mathrm{a}}$ & $3,9^{\mathrm{a}}$ & $4,4^{\mathrm{a}}$ \\
Gosto salgado & $3,0^{\mathrm{a}}$ & $2,8^{\mathrm{a}}$ & $3,8^{\mathrm{a}}$ & $3,6^{\mathrm{a}}$ & $2,9^{\mathrm{a}}$ \\
Gosto azedo & $2,0^{\mathrm{b}}$ & $2,0^{\mathrm{b}}$ & $4,5^{\mathrm{a}}$ & $1,9^{\mathrm{b}}$ & $2,3^{\mathrm{b}}$ \\
Sabor ranço & $1,9^{\mathrm{a}}$ & $1,6^{\mathrm{a}}$ & $3,6^{\mathrm{a}}$ & $1,8^{\mathrm{a}}$ & $2,3^{\mathrm{a}}$ \\
\hline
\end{tabular}

Letras iguais, na mesma linha, não apresentam diferença significativa $(\mathrm{p}>0,05)$. 
cor branca, odor e sabor característicos, sem sabores nem odores estranhos. No entanto, Romero et al. (2001) e Adhikari et al. (2010) descrevem o leite como sendo uma bebida doce, devido à lactose, salgada, devido aos sais inorgânicos, e cremosa ou gordurosa, devido à gordura. Brito et al. (2017) destaca que o leite de boa qualidade apresenta características sensoriais agradáveis, próprias do alimento, como coloração branca e opaca, consistência homogênea, ausência de grumos ou filamentos, odor suave e gosto levemente adocicado. Todas as características descritas por estes autores foram levantadas pelos julgadores neste estudo, no entanto, o que variou foi a intensidade de alguns destes termos sensoriais.

Cabe destacar, que apesar de não ter ocorrido diferença significativa entre as amostras para o sabor a ranço, o leite da marca " $\mathrm{C}$ " apresentou maior intensidade para este termo, além do maior valor para o gosto azedo. Características estas que podem estar relacionadas a maior contagem de microrganismos ou pela presença de enzimas pois, segundo Luck et al. (1980); Bester et al. (1986); Santos (1992); Alfenas (1994); Moura (1997) características como sabor a ranço e gosto ácido no leite podem ser decorrentes da produção de leite pasteurizado a partir de leite refrigerado com alta população de psicrotróficos. Estes autores também destacam que as alterações físico-químicas e bioquímicas que ocorrem no leite estocado a frio são importantes nas características do produto final.

Fatores como o estado sanitário do rebanho, a alimentação e o manejo dos animais, as condições dos equipamentos durante e após a ordenha, a temperatura do produto até o momento do consumo final também podem influenciar no sabor do leite (NELSON et al., 1964; SHIPE et al., 1978; BODYFELT et al., 1988; AZARRA; CAMPBELL, 1992). Aspectos estes que podem justificar as diferenças na intensidade de alguns termos sensoriais, uma vez que são leites de marcas diferentes, portanto submetidos a processos tecnológicos diferentes e oriundos de produtores diferentes.

\section{Aceitação sensorial do leite pasteurizado}

Na Figura 1 e 2, é possível verificar o perfil dos consumidores que realizaram o teste de aceitação sensorial.

Observa-se que a maioria dos participantes foi do sexo feminino (59\%), possuía idade entre 18 a $23(36,4 \%)$ e cursava o técnico (36,4\%). Segundo Osório et al. (2009) a seleção da população de consumidores é ponto crítico desta análise, visto que deve ser representativa, ao menos em sexo e distribuição de idade, da população a estudar.

Verificou-se ainda que a maioria dos participantes consome o leite integral UHT e apenas $13,6 \%$ possui o hábito de consumir o leite pasteurizado integral. Observou-se ainda que apenas $25 \%$ dos participantes consomem leite puro, ou seja, sem a adição de algum sabor.

De acordo com Silva (2008), um dos fatores que interferiu significativamente no mercado de lácteos foi o surgimento e o crescimento do leite longa vida. Segundo o autor, o aumento da demanda de leite UHT é explicado por fatores como os novos hábitos de vida e as facilidades proporcionadas pela embalagem do produto.

Nascimento; Dorr (2010), estudando o perfil dos consumidores de leite, verificaram que alguns dos consumidores de leite UHT afirmam que o consomem devido à praticidade da embalagem, mas acreditam que o leite pasteurizado é realmente "mais parecido com o leite natural" sem ter sido submetido ao processamento.

O baixo consumo do leite pasteurizado e a falta de hábito de consumir leite puro são fatores que justificam a baixa aceitação das diferentes amostras de leite pasteurizado, conforme podemos observar na Tabela 4 . 


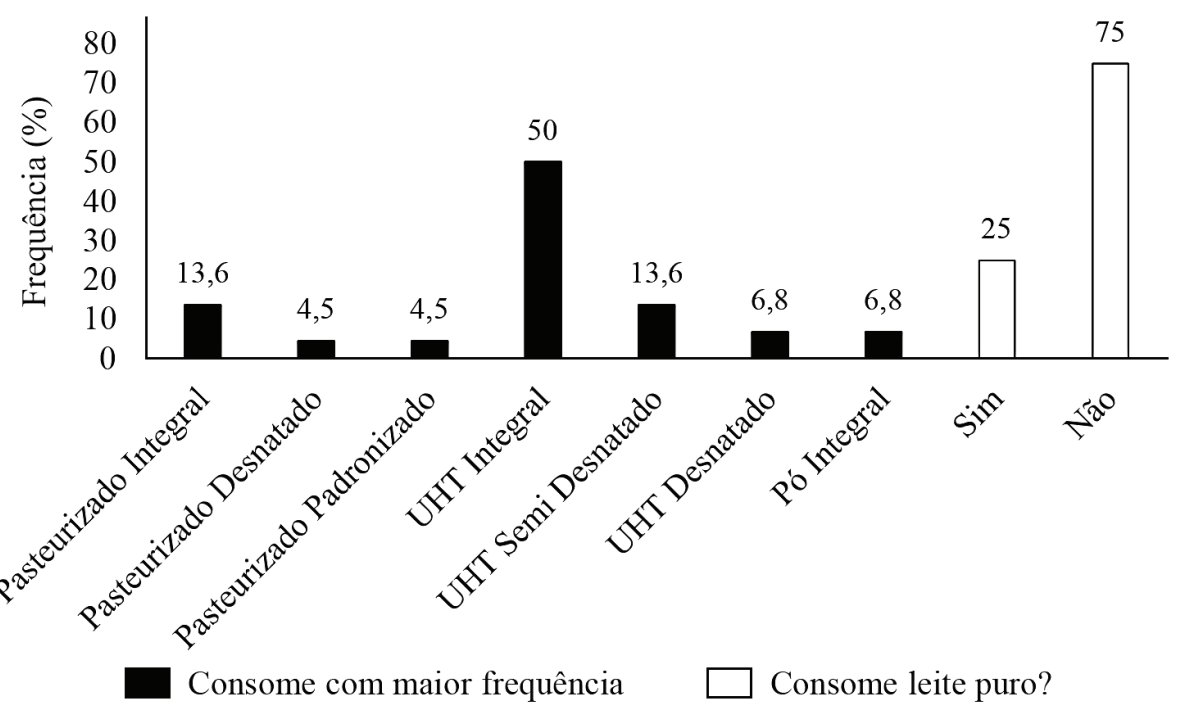

Figura 1 - Perfil dos consumidores que realizaram o teste de aceitação

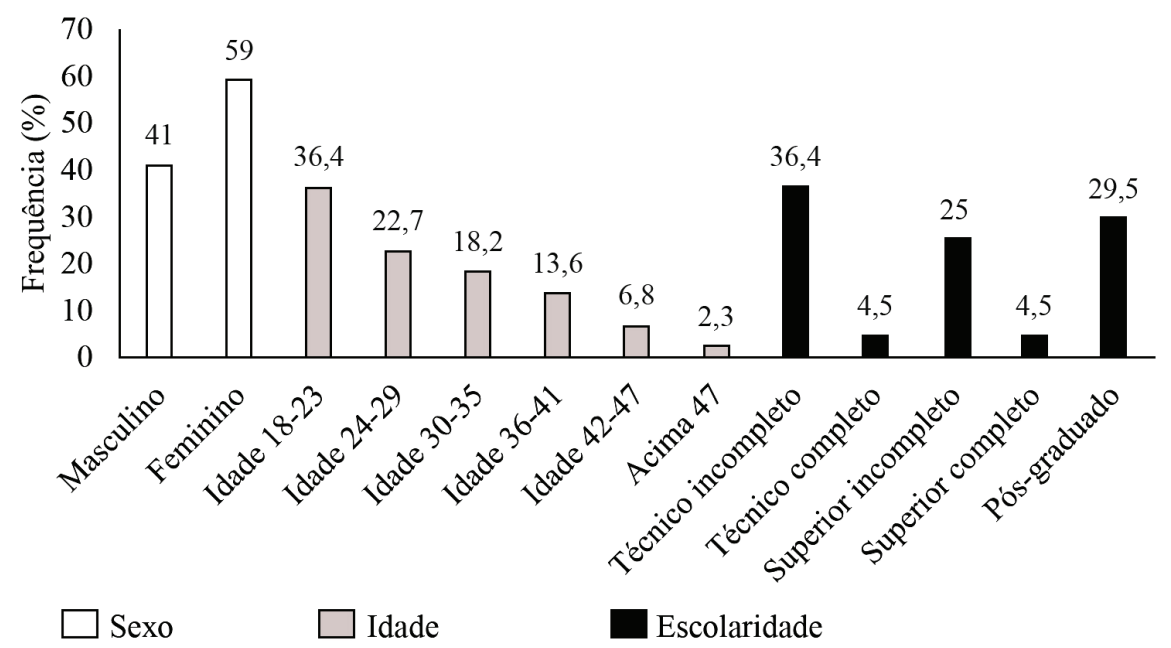

Figura 2 - Tipo e forma de consumo do leite dos participantes do teste sensorial

Tabela 4 - Valores médios de aceitação sensorial do leite pasteurizado integral

\begin{tabular}{cccccc}
\hline & Marca A & Marca B & Marca C & Marca D & Marca E \\
\hline Valor médio & $3,8^{\mathrm{b}}$ & $5,5^{\mathrm{a}}$ & $5,4^{\mathrm{a}}$ & $5,0^{\mathrm{ab}}$ & $5,3^{\mathrm{a}}$ \\
\hline
\end{tabular}

Médias com letras iguais na mesma linha não diferem significativamente entre si $(\mathrm{p}>0,05)$. 
Segundo Font I Furnols et al. (2009), a frequência e a forma de consumo dos produtos influenciam diretamente em sua aceitação sensorial. Também foi possível verificar que o leite da marca $\mathrm{A}$ apresentou a menor aceitação sensorial $(3,8)$, não diferindo significativamente $(\mathrm{p}>0,05)$ apenas do leite da marca $\mathrm{D}(5,0)$. Já as demais amostras não apresentaram diferença significativa entre si ( $p>0,05)$ em relação a aceitação sensorial.

Considerando os resultados obtidos para a aceitação sensorial, é possível verificar que apesar do leite das marcas A e B terem sido submetidos a etapa de homogeneização e os mesmos apresentarem similaridades em relação as respostas do perfil sensorial e instrumental, não apresentaram este mesmo comportamento no que se refere a aceitação sensorial.

Comparando os resultados obtidos na análise do perfil livre e no teste de aceitação, observa-se que o leite da marca $\mathrm{C}$ apresentou maior valor médio para o gosto azedo, diferindo significativamente das outras amostras, e maior intensidade do sabor a ranço. No entanto, não foi a amostra de menor aceitação quando avaliada através da escala hedônica.

A pouca exigência dos consumidores na qualidade sensorial do leite, pode ser corroborado com o estudo desenvolvido por Cortez et al. (2010), os quais realizaram a avaliação sensorial de amostras de leite fraudadas com água e soro de queijo, e verificaram que os provadores detectaram a adição de água no leite, apenas em amostras contendo 30\%. Nos testes com adição de soro de queijo, os julgadores perceberam a adulteração apenas em amostras contendo $45 \%$ de soro.

Bonacina et al.(2015) ao estudar a aceitação sensorial de leite integral UHT (Ultra High Temperature) e as atitudes dos consumidores em relação às embalagens de diferentes marcas do produto, verificaram que vários consumidores, participantes do estudo, destacaram que são pouco exigentes com a qualidade sensorial do leite que consomem e para decisão de compra do produto consideram as características não sensoriais, como por exemplo marca, embalagem, rótulo e preço.

\section{CONCLUSÕES}

Mediante os resultados obtidos, foi possível concluir que as características sensoriais descritas e avaliadas na análise do perfil livre não foram determinantes para definição do leite de maior aceitação. Por isso, é importante a realização de pesquisas visando entender o comportamento do consumidor, para desta forma favorecer as indústrias, nas tomadas de decisão em relação aos produtos fornecidos aos consumidores, os quais possuem seus critérios próprios no que se refere a qualidade sensorial do produto.

Além disso, foi possível observar que a forte correlação obtida entre os resultados sensoriais e instrumentais demonstraram a importância da realização desta relação para a indústria de alimentos e pesquisadores, uma vez que as análises sensoriais descritivas são obtidas com maior demanda de tempo e custo.

\section{REFERÊNCIAS}

ADHIKARI, K. et al. Sensory characteristics of commercial lactose-free milks manufactured in the United States. Food Science and Technology, v. 43, p. 113-118, 2010.

ALFENAS, S. R. C. G. Efeito da temperatura de crescimento e determinação dos parâmetros de resistência ao calor de psicrotróficos acidificantes isolados do leite. 1994. 55p. Dissertação (Mestrado em Microbiologia Agrícola) - Universidade Federal de Viçosa, Viçosa, 1994.

ACEVEDO, N. et al. Water-solids interactions, matrix structural properties and the rate of non-enzymatic browning. Journal of 
Food Engineering, v. 77, n. 4, p. 1108-1115, 2006.

AZARRA, C. D. et al. Off-flavors of Dairy Products. In: CHARALAMBOUS, G. Offflavors in Foods and Beverages. Amsterdam, London, New York, Tokyo: Elsevier, 1992. p. 329-374.

BAKKER J. et al. Tristimulus measurements (CIELab 76) of Port wine color. Vitis, v. 25, p. 67-78, 1986.

BESTER, B. H. et al. Predication of the keeping quality of refrigeranted raw milk. South African Journal Science, Johnnesburg, v. 18, p. 6-11,1986.

BODYFELT, F. W. et al. The Sensory Evaluation of Dairy Products. Nova York: Publication New York, AVI, Van Nostrand Reinhold, 1988. 598 p.

BONACINA, M. S. et al. Aceitação sensorial de leite Integral Ultra High Temperature e atitudes dos consumidores em relação às embalagens de diferentes marcas do produto. Revista do Instituto de Laticínios Cândido Tostes, v. 70, n. 4, p. 216-225, 2015.

BRASIL. Ministério da Agricultura, Pecuária e Abastecimento. Instrução Normativa $n^{\circ}$ 62, de 29 de dezembro de 2011. Aprova o Regulamento Técnico de Produção, Identidade e Qualidade do Leite tipo A, o Regulamento Técnico de Identidade e Qualidade de Leite Cru Refrigerado, o Regulamento Técnico de Identidade e Qualidade de Leite Pasteurizado e o Regulamento Técnico da Coleta de Leite Cru Refrigerado e seu Transporte a Granel, em conformidade com os Anexos desta Instrução Normativa. Diário Oficial da República Federativa do Brasil, 30 dez. 2011. Seção 1, p. 6.
BRITO, M. A. et al. Composição do leite. Agência de Informação Embrapa - Agronegócio do Leite Disponível em:<http:// www.agencia.cnptia.embrapa.br/Agencia8/ AG01/arvore/AG01_128_21720039243. html>. Acesso em: 25 abr. 2017.

CORTEZ, M. A. S. et al. Características físico-químicas e análise sensorial do leite pasteurizado adicionado de água, soro de queijo, soro fisiológico e soro glicosado. Revista do Instituto de Laticínios Cândido Tostes, v. 376, n. 65, p. 18-25, 2010.

DATTATREYA, A. et al. Moderately acidic $\mathrm{pH}$ potentiates browning of sweet whey powder. International Dairy Journal, v. 16, p. 822-828, 2006.

FERREIRA, D. N. Influência do uso de retentados de baixo fator de concentração no rendimento e na qualidade da mussarela de reduzido teor de gordura feita por acidificação direta. 2004. Dissertação (Mestrado em Tecnologia de Alimentos) Programa de Pós-Graduação em Tecnologia de Alimentos, UNICAMP, Campinas, 2004.

FONT I FURNOLS, M. F. et al. Acceptability of lamb fed on pasture, concentrate or combinations of both systems by European consumers. Meat Science, v. 81, n. 1, p. 196202, 2009.

GARCÍA-PÉREZ, F. J. Effect of Orange fiber addition on yogurt color during fermentation and cold storage. Industrial Apllications, v. 30, n. 6, p. 457-463, 2005.

GIGANTE, M. L. Importância da qualidade do leite no processamento de produtos lácteos. In: DURR, J. W., CARVALHO, M. P., SANTOS, M. V. O Compromisso com a Qualidade do Leite. Passo Fundo: Editora UPF, 2004, v. 1, p. 235-254. 
HUNTERLAB. Applications note: CIE L* a* b* color scale. Virginia, 1996. v. 8, n. 7, p. 1-4. 1996.

JING, H.; KITTS, D. D. Chemical and biochemical properties of casein sugar Maillard reaction products. Food and Chemical Toxicology, v. 40, p. 1007-1015, 2002.

KELLY, G. A. The psychology of personal constructs. New York: Norton, 1955. 1082 p.

KITZBERGER, C. S. G. et al. Caracterização sensorial de cafés arábicas de diferentes cultivares produzidos nas mesmas condições edafoclimáticas. Brazilian Journal of Food Technology, v. 6, n. 1, p. 39-48, 2010.

LEÓN K. et al. Color measurement in L*a *b* units from RGB digital images. Food Research International, p. 1084-1091, 2006.

LUCK, H. et al. Shelf life testes on pasteurized milk. South African Journal of Dairy Technology, Johannesburg, v. 12, p. 107-112, 1980.

MACDOUGALL D. B. Colour in food Improving quality. Woodhead Publishing Limited. 2002. 12p.

MINOLTA. K. Precise color communication: color control from feeling to instrumentation. MINOLTA Co. Ltd. Japão, 1994. 49p.

MOURA, C. J. Efeito do resfriamento do leite sobre o rendimento e lipólise do queijo tipo parmesão. 1997. 77 p. Dissertação (Mestrado em Ciência dos Alimentos) - Universidade Federal de Lavras, Lavras, 1997.

NASCIMENTO, A. R.; DORR, A. C. Análise Econômica do Perfil dos Consumidores de Leite em Santa Maria - RS. In: CONGRESSO
DA SOCIEDADE BRASILEIRA DE ECONOMIA ADMINISTRAÇÃO E SOCIOLOGIA RURAL, 48., 2010, Campo Grande. Anais eletrônicos... Campo Grande, 2010. Disponível em: <http://www.sober. org.br/palestra/15/54.pdf $>$ Acesso em: 10 mai. 2017.

NELSON, J. A. et al. Judging Dairy Products. $4^{\mathrm{a}}$ ed. Olsen: Publication Milwaukee, 1964. 486 p.

OSÓRIO, J. C. et al. Características sensoriais da carne. Revista Brasileira de Zootecnia, v. 38, p. 292-300, 2009.

ROMERO, C. et al. Effect of storage temperature on galactose formation in UHT milk. Food Research International, v. 34, p. 389-392. 2001

SANTOS, M. T. M. Efeito do tratamento térmico do leite na qualidade do queijo Minas. 1992. 53p. Dissertação (Mestrado em Ciência e Tecnologia de Alimentos) - Universidade Federal de Viçosa, 1992.

SCHAFFER, E. et al. 1992.Tristimulus colour reflectance measurement of milk and dairy products. Lait, v. 72, n. 4, p. 383-391.

SHIPE, W. F. et al. Off Flavors of Milk: Nomenclature, Standards, and Bibliography. Journal of Dairy Science, Champaign, v. 61, n. 7, p. 855-869, 1978.

SILVA, R. O. P. Evolução das Características do Mercado de Leite. Revista Análises e Indicadores do Agronegócio, v. 3, n. 10, 2008.

SOEIRO, T. N. Indústria de alimentos. Universidade Federal do Espírito Santo, Espírito Santo, 2011. 
STONE, H. S. et al. Sensory evaluation practices. San Diego: Academic Press, 1993. 308 p.

STONE, H. et al. Descriptive Analysis. In: STONE, H. et al. Sensory Evaluation Practices. San Diego: Academic Press, $3^{\mathrm{a}}$ ed. 2004, p. 201-245.

TRONCO, V. M. Manual para Inspeção da Qualidade do Leite. $5^{\mathrm{a}}$ ed. Santa Maria: Editora da UFSM, 2013. p. 95 a 127.

TEIXEIRA, L. V. Análise Sensorial na Indústria de Alimentos. Revista do Instituto de Laticinio Cândido Tostes, n. 366, v. 64, p. 12-21, 2009.

WILLIAMS, A. A. et al. The use of freechoice profiling for the evaluation of comercial ports. Journal of the Science of Food and Agriculture, v. 35, n. 5, p. 558, 1984.
VENTURINI, K. S. et al. Processamento do leite. Boletim Técnico. Universidade Estadual do Espírito Santo, Espírito Santo. 7p. 2007. Disponível em:<http://www.agais.com/ telomc/b022_processamento_bovinoleite. pdf $>$ Acesso em: 30 mai. 2017.

VILLANUEVA, N. D. M. et al. Performance of the hybrid hedonic scale as compared to the traditional hedonic, self-adjusting and ranking scales. Food Quality and Preference, Barking, v. 16, n. 8, p. 691-703, 2005.

VERRUMA-BERNARDI, M. R. et al. Uso do Perfil Livre na análise de queijo Mozarella de leite de búfula elaborado pelos métodos tradicionais e de acidificação direta. In: ALMEIDA, T. C. A. et al. Avanços em análise sensorial. São Paulo: Varela, 1999. p. 261-281. 